\title{
Distribution Management by Means of Cutoff Order Size: A Case Study
}

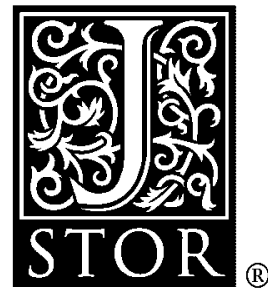

\author{
R. Nass; R. Dekker; W. van Sonderen-Huisman
}

The Journal of the Operational Research Society, Vol. 48, No. 11 (Nov., 1997), 1057-1064.

Stable URL:

http://links.jstor.org/sici?sici=0160-5682\%28199711\%2948\%3A11\%3C1057\%3ADMBMOC\%3E2.0.CO\%3B2-E

The Journal of the Operational Research Society is currently published by Operational Research Society.

Your use of the JSTOR archive indicates your acceptance of JSTOR's Terms and Conditions of Use, available at http://www.jstor.org/about/terms.html. JSTOR's Terms and Conditions of Use provides, in part, that unless you have obtained prior permission, you may not download an entire issue of a journal or multiple copies of articles, and you may use content in the JSTOR archive only for your personal, non-commercial use.

Please contact the publisher regarding any further use of this work. Publisher contact information may be obtained at http://www.jstor.org/journals/ors.html.

Each copy of any part of a JSTOR transmission must contain the same copyright notice that appears on the screen or printed page of such transmission.

JSTOR is an independent not-for-profit organization dedicated to creating and preserving a digital archive of scholarly journals. For more information regarding JSTOR, please contact support@ jstor.org. 


\title{
Distribution management by means of cutoff order size: a case study
}

\author{
R Nass, R Dekker and W van Sonderen-Huisman \\ Erasmus University, Rotterdam
}

We present a case study on physical distribution management for a production company in Western Europe. The company delivers finished goods both from distribution centres and directly from plants to its customers. The lead time from distribution centres is shorter, but higher costs are involved. The choice for delivery of an individual order is based on the so-called stockmix and cutoff order size. The stockmix is the set of products stocked at the distribution centre, which for efficiency reasons is restricted. Orders smaller than the cutoff order size are delivered from the distribution centre closest to the customer, provided that the product ordered is present in its stockmix. Otherwise they are delivered from the production plant that makes the product. In this paper we develop methods to determine both the stockmix and the cutoff order size for each distribution centre. The objective considered is the minimisation of distribution and handling costs subject to service constraints.

Keywords: distribution; transport; simulation; heuristics

\section{Introduction}

The company considered produces a variety of self-adhesive materials that are sold in roll form throughout Western-Europe. Products are shipped to industrial customers directly from three production plants (PPs) and from six distribution centres (DCs) in Western Europe. The lead time for a delivery from a DC is shorter than from a PP, as the product is then delivered from stock, while in a PP some production stages still need to be gone through, for example coating. Calculations, however, showed that in most cases delivery from a PP is cheaper, due to lower handling costs. As service reliability is considered to be a key factor for the company, management undertakes regular studies to ensure fast delivery to customers while keeping distribution costs as low as possible. Hence, one of the main issues facing the company is to decide which orders to deliver from a DC and which from a PP. The company uses two means of control. The first is the stockmix, namely the set of products stocked in a DC. From the 10000 different products only some 300 have a turnover large enough to be in the DC-stockmix. From this number a further selection is made, based on the particular demands of the country. For most other products, components are stocked at the PPs, which need some combination and finishing before they can be delivered. Specialised products are made to order at the plants and have a much longer lead time. The goal of the second form of control is to reduce

Correspondence: $\operatorname{Dr} R$ Dekker, Erasmus University, Rotterdam, The Netherlands. handling costs. The company applies a minimum order quantity above which orders are delivered directly from the $\mathrm{PP}$, even if the product is stocked in the DC. This quantity is called the cutoff order size. Each DC has a specific stockmix and cutoff order size; both remain the same for some time. Together they determine which orders are supplied from a DC and which from a PP. This is achieved through the order information system, which is used all over Europe, in which all the stockmixes, the inventory levels of all products in the DCs and the cut-off order sizes are stored. Upon order entry, the customer gets information on the intended delivery date of his order. After acceptance, the order is assigned to the appropriate DC or PP, according to the prevailing stockmix and cutoff order size.

\section{Problem details}

In Figure 1 the distribution network and the locations of the DCs and PPs are given. Each customer order specifies the products and amount ordered. The width of the roll ordered may be any value over $50 \mathrm{~mm}$; the length must be a multiple of $500 \mathrm{~m}$. If the width ordered is not the same as that of the standard width, the roll must be cut to the desired width. This must be done for about half of the orders. Cutting can be done in the PPs as well as in the DCs. The cutting capacities are the only factors in our study which restrict the daily throughput volume in each location.

The management asked us to develop methods to determine the stockmix and the break quantities. The methods needed to be used on a regular basis because of market dynamics the optimal stockmixes and cutoff order sizes 


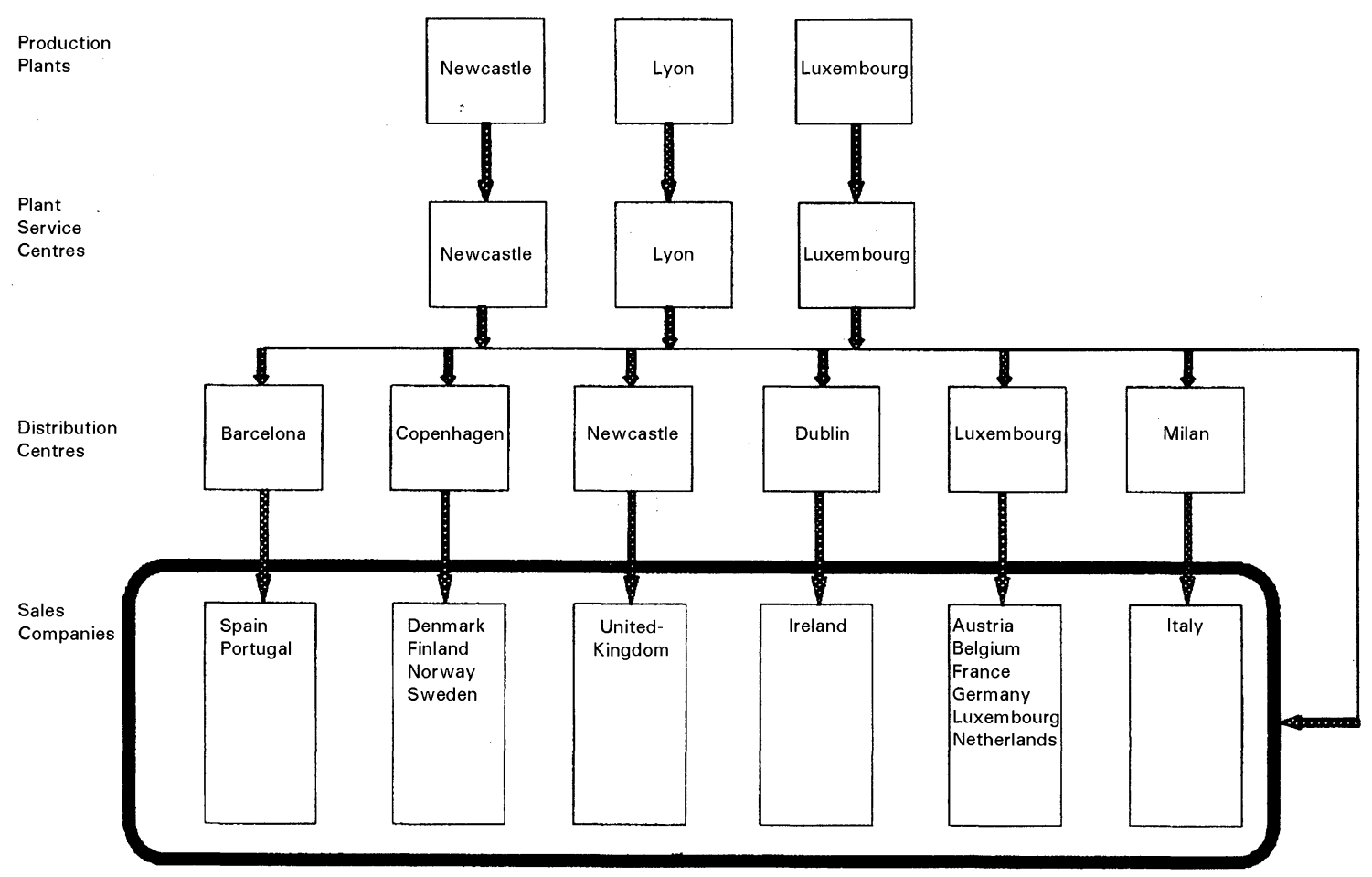

Figure 1 The distribution network.

may change quite frequently. In this study we focus on cost minimisation under certain service requirements. The costs considered include the transportation costs and the extra handling and packaging costs for orders shipped via a DC. Inventory costs are ignored because their impact is judged to be low due to the high inventory turnover and the limited number of products in the stockmix. The two service requirements considered are speed of delivery and reliability. As delivery through a DC is substantially faster (normally two to three days between order receipt and delivery to the customer) than through a PP (about two weeks), the first requirement is modelled as a minimal percentage of orders shipped via a DC. Service reliability is measured as the maximal percentage of orders shipped too late as a result of insufficient cutting capacity. Order sizes are measured in this investigation in $\mathrm{m}^{2}$ (width times length) due to the customers needs as well as in kilograms due to the transport tariff structures. For transportation purposes the products are equivalent. There is a fixed conversion factor between volume and weight.

\section{Transport costs}

In the distribution system there are three types of transport:
A. From PP to customer: direct transport
B. From PP to DC: stock replenishments skip.
C. From DC to customer: regional transport points.

For each type of transport the company uses various transport companies, each with their own tariff structure. Due to changing market conditions, the transport companies and the tariff structures vary over time. Therefore we selected for each type of transport the most common tariff structure, which is described below.

\section{A. From PP to customer: direct transport}

Each day all direct orders are collected from each PP by a transport company, which takes it to its hub and from there through its network to the customers. For each combination of a PP and a destination country there is a basic rate per kilogram, independent of the exact route length to a customer in that country. On top of this cost rate is a surcharge. The actual cost per kilogram for a direct order on a certain day is the basic rate divided by the average degree of load of all the direct vehicles loaded at that PP that day

$$
\mathrm{DT}_{p, d, t}=\frac{\text { rate }_{p, d}}{\operatorname{load}_{p, t} / \mathrm{capa}_{p, t}}
$$

with

$\mathrm{DT}_{p, d, t}=$ actual cost per $\mathrm{kg}$ on day $t$ for direct transport from PP $p$ to country $d$.

rate $_{p, d}=$ basic rate per $\mathrm{kg}$ for direct transport from PP $p$ to country $d$. 
$\operatorname{load}_{p, t}=$ total load in all the direct vehicles leaving PP $p$ on day $t$.

capa $_{p, t}=$ total capacity of all the direct vehicles leaving PP $p$ on day $t$.

Hence the direct transport costs for an individual order depends on the total volume shipped directly from the PP on that day, which varies from day to day. It depends indirectly on the stockmix and the cutoff order size for all DCs, the factors which have to be determined in our study. Note that the cost per $\mathrm{kg}$ does not always decrease if the volume increases: if one more truck is needed the cost increases.

\section{B. From PP to DC: stock replenishments}

For this type of transport time is not crucial; a system of full truck loads is used. Replenishments for a certain DC leave as soon as there is need for a full truck load. So the cost per kilogram of the PP-DC transport of an order delivered via a DC is fixed for each PP-DC combination.

\section{From DC to customer: regional transport}

Regional transport may cover several countries (for example from the DC Luxembourg to France, Germany, etc). Each day the orders packed at a DC are shipped to the customers. The cost rate per kilogram depends on the order size as well as the destination; the cost rate decreases as the order size increases. There are several weight classes. In each class there are basic rates per kilogram per DCcountry combination.

\section{Related research}

Most distribution literature focuses either on strategic issues, such as locating DCs, or on operational issues as vehicle routing, where as our study deals with tactical optimisation. There are a few papers which deal with a kind of cutoff order size concept. Sussams ${ }^{1}$ considers the choice between direct and indirect transport for a given customer. Sussams determines a minimum order size for direct transport, which is comparable to our cutoff order size. The transport costs are specified for each order (no consolidation) and the tactical problem, of setting a cutoff order size for a whole group of customers, is not considered. Hollier $e t a l^{2}$ and Dekker $e t a l^{3}$ consider a cutoff order size (also called cutoff transaction size or break quantity) in an inventory context and show that inventory costs can be reduced substantially by using the cutoff order size concept. Hollier $e t a l^{2}$ consider a single stocking point, while Dekker et $a l^{3}$ consider a one warehouse $n$-retailer system. Other studies dealing with the choice between direct and indirect transport are from Daganzo, ${ }^{4}$ Kasturi et $a l^{6}$ and Hall. ${ }^{5}$ All these studies treat the problem in a deterministic setting, with complete knowledge of all future flows and with simplified cost functions. In those cases good optimisation results can be obtained. Daganzo ${ }^{4}$ also considers the option to distribute directly from a plant or from a distribution centre. He assumes fixed distribution costs per $\mathrm{kg}$ for each PP- or DC-customer combination. These costs mainly depend on the locations of the plants and distribution centres. Distribution via a DC involves higher handling costs. This problem can be formulated as a linear program with variables indicating the amount supplied per DC to a customer. Due to the assumptions it has a $0-1$ solution. Our case has a different cost structure. Moreover, capacity constraints further complicate our problem. Kasturi et $a l^{6}$ consider consumer rebates if direct transportation is accepted. The company can choose the rebates such that its distribution costs are minimised. Again only a simplified cost structure is assumed. Hall ${ }^{5}$ studies direct versus terminal freight routing on a network with concave costs and with many origins and few destinations. For this case a simple and efficient algorithm is derived. Again, our transport costs are not concave. We note that the cutoff order size and the stockmix concepts are especially useful for the stochastic case, in which orders arrive one at a time and an immediate decision on the routing of each order has to be made.

\section{Analysis}

The management preferred to have a PC-based optimisation program in which service requirements could be entered and historical order data be used. The outcomes would then be implemented in the current period. The order data could be down loaded from the sales information system. The following data was available on the orders (numbered 1 to $N$ ): quantity of order $i$ in kilograms $Q_{i}$, order entry day $e t_{i}$, country of destination $d_{i}$, the $\mathrm{DC} d c_{i}$ and the PP $p_{i}$ from which the order can be delivered. The objective function of the distribution problem can be formulated as

$$
\begin{aligned}
\min C= & \sum_{i} Q_{i}\left(1-x_{i}\right) \mathrm{DT}_{p_{i}, d_{i}, t_{i}}+\sum_{i} Q_{i} x_{i} \mathrm{SR}_{p_{i}, d c_{i}} \\
& +\sum_{i} Q_{i} x_{i} \mathrm{RT}_{d c_{i}, d_{i}, q_{i}}
\end{aligned}
$$

with

$x_{i} \quad=$ decision variable which is 1 when order $i$ is delivered via a DC and 0 otherwise

$\mathrm{DT}_{p_{i}, d_{i}, t_{i}}=$ direct transport cost per $\mathrm{kg}$ from $\mathrm{PP} p_{i}$ to country of destination $d_{i}$ on day $t_{i}$.

$t_{i} \quad=$ the shipping day of order $i$, namely the day order $i$ leaves either PP $p_{i}$ (direct transport) or $\mathrm{DC} d c_{i}$ (regional transport).

$\begin{aligned} \mathrm{SR}_{p_{i}}, d c_{i} & =\text { stock replenishment cost per kg from PP } p_{i} \text { to } \\ & \mathrm{DC} d c_{i}\end{aligned}$ 


\section{$\mathrm{RT}_{d c_{i}, d_{i}, q_{i}}=$ regional transport cost per $\mathrm{kg}$ (including hand- ling cost) or an order from DC $d c_{i}$ to country of destination $d_{i}$ and in weight class $q_{i}$}

Note that $x_{i}$ is one only if the product requested is in the stockmix and the amount ordered $Q_{i}$, is below the cutoff order size $\mathrm{CS}_{d c}$, used in that DC. The following service requirements were formulated: a fraction $\alpha$ of all orders should be delivered via DCs: $1 / N \sum_{i} x_{i} \geqslant \alpha$ and a fraction $\delta$ of all orders should be shipped in one day from the DCs: $1 / N \sum_{i} x_{i} y_{i} \geqslant \delta$, where the variable $y_{i}$ is 1 (and 0 otherwise) if the order is shipped in time from the DC. Because in each PP there is enough cutting capacity, direct orders can be left out.

Before developing a solution method we first analysed the data to get a better understanding of the problem: what determines the total cost level? Which orders must be shipped directly and which orders via a DC? There is no cost difference between the various products; the origindestination combinations and the order pattern of a certain product determine the costs needed to ship the orders of that product. We can calculate in advance the cost of a certain order when it is shipped via a DC; however we cannot calculate the cost when this order is shipped directly because this also depends on other directly shipped orders from the same PP. So we cannot compare for a certain order the cost difference in shipping directly or via a DC. From the tariff tables it appears that direct shipping is in general cheaper than shipping via a DC.

\section{Decomposition of the problem into subproblems}

The dependence of the direct transport cost on the cutoff order sizes in all DCs and the stockmixes calls for an integrated optimisation model. However, several problems exist with such a model in our study. First, a major practical problem arises from the large number of products that can be stocked. Each product requires for each DC the use of a $0 / 1$ stock mix variable to define whether it is stocked or not in that DC. Each DC requires the computation of one CS variable. The 300 products and the six DCs give rise to 1800 stockmix variables and six CS variables. Secondly, the complex structure of the transportation costs makes the objective function nonlinear. Another disadvantage of an integrated model is the acceptance by the management of the resulting stock lists and cutoff order sizes. This is because the optimization model provides no insight about why this specific solution results in the lowest costs. Hence other methods needed to be tested.

Therefore, we tried decomposing the problem into subproblems. The most logical decomposition is a subproblem for each DC but, as argued before, this is impossible. We therefore applied a different decomposition: we solve the problem in two stages. In the first stage we determine the stockmix for each DC. In the second stage we deter- mine a good set of cutoff order sizes, based on the selected stockmixes.

\section{A simulation program}

In each stage we use a simulation program to calculate total costs and service performance. The program uses information about all orders in the preceding period. Furthermore, the program requires transport tariffs, cutting capacities and finally for each DC the list of stocked products and the cutoff order size. The output consists of the total transport costs for replenishment, regional and direct shipments, the number of orders that were shipped from each location and the number of orders that were shipped too late.

Delays in shipping are caused by a lack of cutting capacity in a location. In practice orders are cut using a first-in-first-out policy. To prevent service performance from dropping below target, locations that lack service capacity are frequently forced to work overtime. We could not model that in our simulation program since no data was available on the overtime policies. To reflect the positive effect of overtime, we used the last-in-first-out (LIFO) policy in the simulation program. As this policy maximises the number or orders cut in time, it gives about the same effect as overtime (the number of days an order is delivered too late does not play a role in the study).

\section{Stage one: determination of the stockmix}

The determination of the number of products to be stocked in a DC is a difficult problem which requires a modelling of the lead time preferences of customers. It was left out of this study; we simply used the present number ( 50 per DC). As the products have the same transportation characteristics, we focused our attention on the order frequency and order size distribution. We started therefore by determining the criteria on which the decision to stock a product should be based.

Firstly we note that the service objectives are formulated in terms of number of orders. The service objectives suggest that one should stock the products which are ordered most often. Examining the stocking decision more closely we find a paradoxical situation. Delivering orders via DCs is more expensive than delivering directly, so the service objectives are best met with shipping small orders via the DCs. This also reduces the workload for the cutting machines in the DCs. However, the cost structure for regional transport, namely decreasing cost per kilogram as the order size increases, makes it worthwhile to stock those 50 products that are ordered mostly in large quantities. Hence we tested the following three index policies: stock those products with largest number of orders (i) below the average order size (over all products), (ii) above and (iii) with any size. 
Stage two: optimising the cutoff order sizes

A simple heuristic is to limit the number of possible choices beforehand and to do an enumerative search over this set. From the order pattern it is obvious that most ordered quantities fall somewhere in the range from $1000 \mathrm{~m}^{2}$ to $10000 \mathrm{~m}^{2}$. Furthermore, trials without the service constraints have shown that the most advantageous cutoff order sizes are either near zero or near the maximum ordered quantity, namely shipping everything via the DC. It is the service constraints that keep them away from these extremes. The most important DCs have another property: when their cutoff order size is below a certain value it becomes impossible to obtain the required percentage of total orders shipped via the DCs. Therefore we considered for each DC the following cutoff order sizes: $0,1,3,6,10$, 17,50 and 100 (in $1000 \mathrm{~m}^{2}$ ). To reduce the total number of combinations even further we took a random selection of some 1432 combinations of cutoff order sizes for each DC, in such a way that for each DC all possible values would occur.

A second method (called estimated cost function) uses the idea of approximating the total costs by a simple function of the cutoff order sizes and to optimise this function rather than the one obtained by the simulation, (similar to the response surface method for simulation optimisation). To obtain such a function we used the data and results considered in the enumeration. The first trial was to fit a linear function by ordinary least squares to this data set, but this yielded a poor fit $\left(R^{2}=0.575\right)$. We examined the data set more closely to obtain a global idea about the shape of the cost function. The function appeared to have its minima at both ends of the range of cutoff order sizes. Hence we tried the following convex function:

$$
C=\beta_{0}+\sum_{d c} \beta_{d c} \mathrm{CS}_{d c}+\sum_{d c} \gamma_{d c} \sqrt{\mathrm{CS}_{d c}\left(\mathrm{CS}_{\max }-\mathrm{CS}_{d c}\right)}
$$

with

$\mathrm{CS}_{d c}=$ the cutoff order size in DC $d c$

$\mathrm{CS}_{\max }=$ the maximum value for the cutoff order size $\left(100000 \mathrm{~m}^{2}\right)$

and $\beta_{0}, \beta_{d c}, \gamma_{d c}$ parameters to be estimated.

The regression showed that this function fits the data rather well $\left(R^{2}=0.93\right)$. The resulting function was optimised using a nonlinear programming solver.

The third method (called 80\% load) is based upon the additional assumption that the trucks for direct transport have a fixed load. Although the actual loads vary substantially, we used the average value, being $80 \%$ of the maximum capacity. The transportation costs can now be computed for each order separately as soon as it has been determined whether the order will be shipped from a PP or from a DC. The total cost function is reduced to a sum over some simple multiplications:

$$
\begin{aligned}
\min C= & \sum_{i} Q_{i}\left(1-x_{i}\right) \mathrm{DNEW}_{p_{i}, d_{i}} \\
& +\sum_{i} Q_{i} x_{i} \mathrm{SR}_{p_{i}, d c_{i}}+\sum_{i} Q_{i} x_{i} \mathrm{RT}_{d c_{i}, d_{i}, q_{i}}
\end{aligned}
$$

with

$$
\begin{aligned}
\mathrm{DNEW}_{p_{i}, d_{i}}= & \text { direct transport cost per } \mathrm{kg} \text { from PP } p_{i} \text { to } \\
& \text { country of destination } d_{i} \text {, assuming } 80 \% \\
& \text { load in the direct transport trucks. }
\end{aligned}
$$

In this case we optimise each DC separately.

In Figure 2 an overview of the three cutoff order size optimisation methods is given.

\section{Implementation}

The simulation program was coded in Turbo Pascal. The output was then imported into the spreadsheet Excel in which the optimisation took place using the associated nonlinear programming (NLP) solver. Although we simplified the objective function in two of the three CS methods we still have to consider the constraints. In the enumeration method we can simply leave out those combinations in which the service constraints are not met. For the two other methods we replaced the constraints on the reliability of service by deterministic constraints on the total throughput for each DC in an approximate way. As the NLP solver often got stuck in local minima, it was restarted many times. The best 100 solutions were selected and entered in the simulation program to check whether they met the original service constraints. Finally, for both methods the best solution was chosen. The simulation of the 1432 combinations took $3 \mathrm{~h}$ on a $486 / 33 \mathrm{MHz}$ PC. The optimisation by both the estimated cost function and the $80 \%$ load function took about $1 \mathrm{~h}$.

\section{Tests and results}

We did extensive tests on the methods using real order data over a representative period. Here we show some results which are indicative of the general outcomes. We first deal with the stockmix determination and then we evaluate the three CS optimisation methods. Finally, we compare the currently used stockmix and cutoff order sizes with the outcomes of the methods presented here. The following basic figures apply to all three parts of the results. The minimum percentage of orders that should be shipped via the DCs was set at $74 \%$, while the minimum percentage of orders shipped on time was set at $95 \%$. The cutoff order sizes could assume any value between 0 and $100000 \mathrm{~m}^{2}$. 


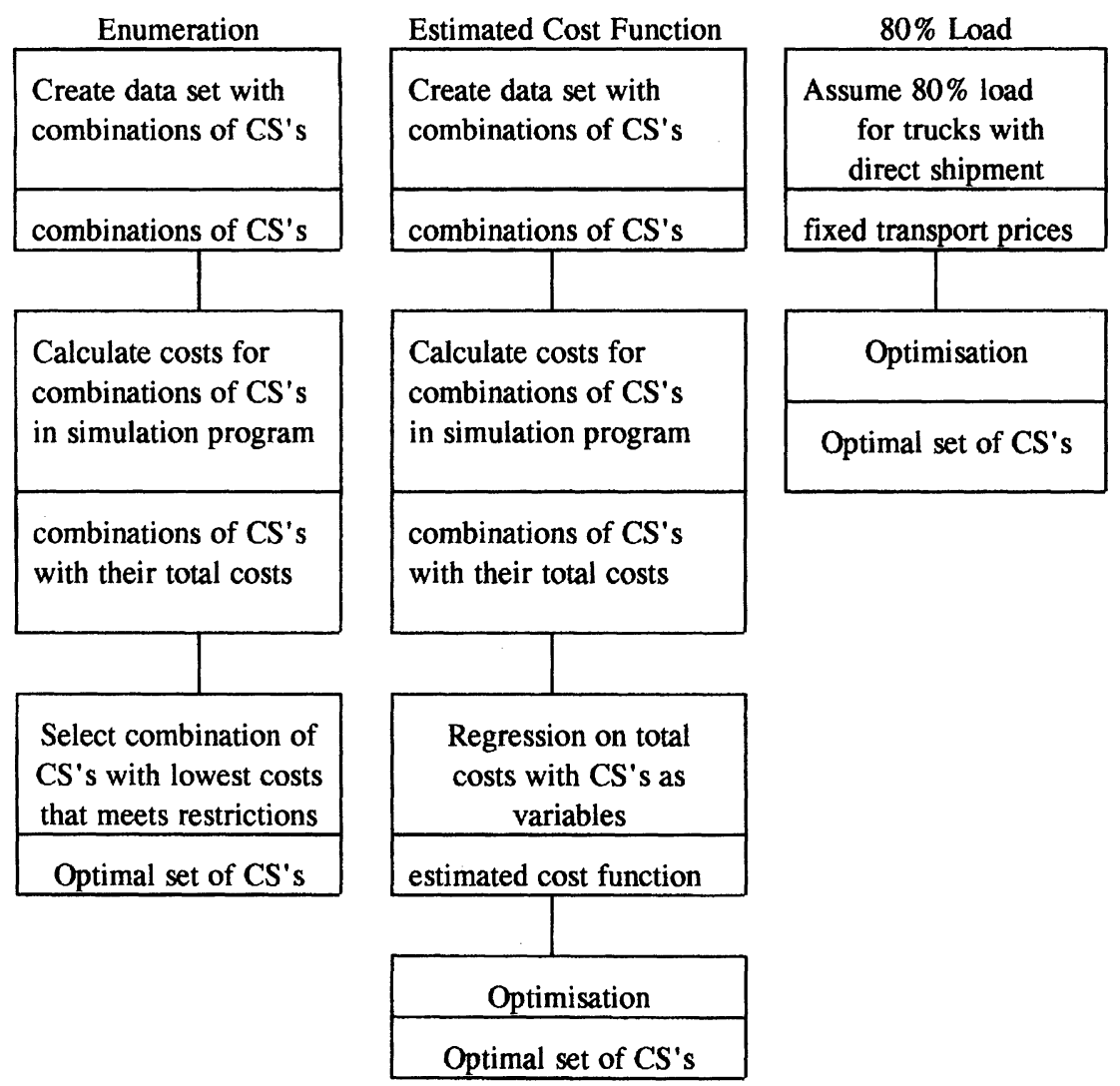

Figure 2 Overview of the three CS optimisation methods.

Which stockmix performs best?

The results in this and the next part were obtained by using both the estimated cost function and enumeration method to find the cutoff order sizes; we used the best solution. In Table 1 we present the results for the three different stockmixes: stocking the products with most frequent small orders, all orders and large orders respectively. The results clearly show which effect dominates: the diminishing costs per kilogram for delivering large orders via the DCs are less important than the lower costs for delivery orders direct from the plants. Stockmixes containing those products that are ordered most often in large quantities give rise to higher overall costs. This means that extra costs for shipping more volume via the DCs dominate the reduced costs per kilogram for regional transport. This amounts to $10 \%$ higher costs compared with the other stockmixes. The cost difference between the stockmixes based upon small and all orders is negligible (5.76 vs 5.75 million Dfl).

\section{Which CS optimization method to use?}

Table 2 shows the results for the three methods. We used a stockmix that contains the most ordered (regardless of the
Table 1 Results for the three different stockmixes

\begin{tabular}{lccc}
\hline & \multicolumn{3}{c}{ Stock most frequent } \\
\cline { 2 - 4 } & Small orders & All orders & Large orders \\
\hline DC & \multicolumn{2}{c}{ cutoff order size (in $1000 \mathrm{~m}^{2}$ ) } \\
Barcelona & 100 & 40 & 17 \\
Copenhagen & 9 & 100 & 100 \\
Newcastle & 4 & 4 & 6 \\
Dublin & 10 & 10 & 50 \\
Luxembourg & 5 & 4 & 17 \\
Milan & 10 & 13 & 100 \\
\% Shipped via DC & 74.6 & 74.7 & 74.6 \\
\% Shipped too late & 4.0 & 4.7 & 3.2 \\
Costs summary (in Dfl & $1000000)$ & & \\
DC activities & & & \\
Packing & 0.45 & 0.48 & 0.69 \\
Handling & 0.35 & 0.38 & 0.54 \\
Transport & & & \\
Replenishments & 0.76 & 0.87 & 1.08 \\
Regional & 1.52 & 1.58 & 2.08 \\
Direct & 2.68 & 2.44 & 1.91 \\
Total & 5.76 & 5.75 & 6.30 \\
\hline
\end{tabular}


Table 2 Results for the three CS optimisation methods

\begin{tabular}{lccc}
\hline & $80 \%$ Load & $\begin{array}{c}\text { Estimated cost } \\
\text { function }\end{array}$ & Enumeration \\
\hline DC & \multicolumn{3}{c}{ cutoff order size (in $1000 \mathrm{~m}^{2}$ ) } \\
Barcelona & 45 & 45 & 50 \\
Copenhagen & 9 & 9 & 17 \\
Newcastle & 4 & 4 & 3 \\
Dublin & 10 & 10 & 50 \\
Luxemburg & 5 & 5 & 6 \\
Milan & 10 & 10 & 6 \\
\% Shipped via DC & 74.6 & 74.6 & 75.1 \\
\% Shipped too late & 4.0 & 4.0 & 3.0 \\
Costs summary (in Dfl & $1000000)$ & & \\
DC activities & & & \\
Packing & 0.45 & 0.45 & 0.47 \\
Handling & 0.35 & 0.35 & 0.37 \\
Transportation & & & \\
Replenishments & 0.76 & 0.76 & 0.82 \\
Regional & 1.52 & 1.52 & 1.53 \\
Direct & 2.68 & 2.68 & 2.62 \\
Total & 5.76 & 5.76 & 5.81 \\
\hline
\end{tabular}

quantity ordered) products. The results show that the solutions presented by the $80 \%$ load and the estimated cost function methods are equal. The results for the enumeration method are worse as far as total costs are concerned, but the service performance is slightly better.

Based on many experiments, we decided to use a combination of the enumeration and estimated cost function method. The estimated cost function method performs in all cases equally well or better than the $80 \%$ load method. The enumeration method is less consistent. In some cases it provides the best solution, while in other cases results are worse. However, both the estimated cost function and the enumeration method are based upon a data set, which can be used for both methods. The most time consuming part of both methods is the construction of this data set, but once this set is available the time needed to obtain the final solution is relatively short.

We noted that the cutoff order sizes found may lack robustness, namely a slight change in the cost function or in the constraint may give quite different cutoff order sizes. This can be observed in the comparison of the methods. This effect seems more dramatic than it is, since the tail of the order size distribution is quite thin. Hence a change of a CS from say 10000 to $50000 \mathrm{~m}^{2}$ affects few orders.

\section{Currently used stockmix and cutoff order sizes compared to our method}

After the final selection of the methods we tested their effect on the present situation. Table 3 shows the results for three cases. The first column shows the results when the actual stockmixes and cutoff order sizes are used. The
Table 3 Comparison of actual and optimized CSs and stockmixes

\begin{tabular}{lccc}
\hline & $\begin{array}{c}\text { Actual } \\
\text { stockmix, } \\
\text { actual CSs }\end{array}$ & $\begin{array}{c}\text { Actual } \\
\text { stockmix } \\
\text { optimized CSs }\end{array}$ & $\begin{array}{c}\text { Stock most } \\
\text { frequent } \\
\text { orders (all), } \\
\text { optimized CSs }\end{array}$ \\
\hline DC & cutoff order size (in $1000 \mathrm{~m}^{2}$ ) \\
Barcelona & 24 & 100 & 10 \\
Copenhagen & 21 & 100 & 11 \\
Newcastle & 10 & 9 & 0 \\
Dublin & 10 & 15 & 10 \\
Luxembourg & 20 & 100 & 5 \\
Milan & 21 & 14 & 5 \\
\% Shipped via DC & 63.5 & 63.6 & 63.7 \\
\% Shipped too late & 3.2 & 2.4 & 3.7 \\
Costs summary (in Dfl & $1000000)$ & & \\
DC activities & & & \\
$\quad$ Packing & 0.52 & 0.54 & 0.37 \\
$\quad$ Handling & 0.41 & 0.43 & 0.29 \\
Transport & & & \\
$\quad$ Replenishments & 0.94 & 1.02 & 0.65 \\
Regional & 1.63 & 1.65 & 1.22 \\
Direct & 2.35 & 2.19 & 3.03 \\
Total & 5.85 & 5.84 & 5.56 \\
\hline
\end{tabular}

second column shows a cutoff order size solution determined by our method, using actual stockmixes. The last column shows the results for the case in which we have used our method to determine both the stockmixes and the cutoff order sizes. For the latter two cases we used the restriction that the percentage of orders shipped via the DCs should at least be equal to the percentage obtained in the first case.

The first two columns show that the currently used stockmixes do not leave much room for improvement. The actual stockmixes only allow minor adjustments to the cutoff order sizes, as otherwise the restriction of the fraction of orders shipped via a DC is not met. The last column shows that a considerable reduction in the total costs can be obtained (5\%) when the stockmixes are based upon the most frequently ordered products. Alternatively, comparing the first column of Table 3 (actual situation) with Table 2 shows that an increase of delivery through a DC from $63-75 \%$ can be obtained against $2 \%$ lower costs!

\section{Conclusions}

The cutoff order size and stockmix are interesting concepts to optimise a company's physical distribution. In our case it was difficult to optimise them because of the complex transport cost structures. Using the heuristics presented in this paper, the company can regularly determine their stockmixes and cutoff order sizes. Based on the results, the company is convinced that our study was worthwhile. 
During our study, some cutoff order sizes were changed along the lines we suggested.

Acknowledgments - The authors thank Jan Dokter, logistic director of the company, for his assistance during the study, Albert Wagelmans, Kerry Malone and the referees for useful comments.

\section{References}

1 Sussams JE (1992). Logistics Modelling. Pitman Publishing, London.

2 Hollier RH, Mak KL and Lam CL (1995).Continuous review $(\mathrm{s}, \mathrm{S})$ policies for inventory systems incorporating a cutoff transaction size. Int J Prod Res 33: 2855-2865.
3 Dekker R, Kleijn MJ and Kok AG de (1996). The break quantity rule in a 1-warehouse, n-retailers distribution system. Preprints 9th International Working Seminar on Production Economics. IGLS, Austria, pp 301-312.

4 Daganzo CF (1991). Logistics System Analysis. Lecture Notes in Economical \& Mathematical Systems, no. 361. SpringerVerlag, Berlin.

5 Hall RW (1987). Direct versus terminal freight routing on a network with concave costs. Trans Res 21B: 287-298.

6 Kasturi Rangan V and Jaikumar R (1991). Integrating distribution strategy and tactics: a model and an application. Mgmt Sci 37: 1377-1389.

Received February 1995; accepted June 1997 after two revisions 